\title{
The Enigma in Management of Complicated Foreign Body Ingestion Impacted Distal to Oesophagus: A Case Series and Literature Review
}

\section{Raymond Zhun Ming Lim}

Hospital Sultanah Aminah https://orcid.org/0000-0002-8243-9812

\section{Addy Aun Wei Ang}

Hospital Sultanah Aminah

Jih Huei Tan ( $\square$ huei_87@hotmail.com )

Hospital Sultanah Aminah https://orcid.org/0000-0001-9833-5164

\section{Ee Peng Lee}

Hospital Sultanah Aminah

Jun Loong Chiew

Hospital Sultanah Aminah

Henry Chor Lip Tan

Hospital Sultanah Aminah

\section{Keywords:}

Posted Date: September 25th, 2020

DOl: https://doi.org/10.21203/rs.3.rs-80657/v1

License: (c) (1) This work is licensed under a Creative Commons Attribution 4.0 International License. Read Full License 


\section{Abstract}

The authors have requested that this preprint be removed from Research Square. 